\title{
Brain Computer Interfaces as Intelligent Sensors for Enhancing Human-Computer Interaction
}

\author{
Mannes Poel \\ Human Media Interaction \\ University of Twente \\ Enschede, The Netherlands \\ m.poel@utwente.nl
}

\author{
Femke Nijboer \\ Human Media Interaction \\ University of Twente \\ Enschede, The Netherlands \\ femke.nijboer@utwente.nl
}

\author{
Egon L. van den Broek \\ Media and Network Services \\ TNO \\ Delft, The Netherlands \\ vandenbroek@acm.org
}

\author{
Stephen Fairclough \\ School of Natural Sciences \\ and Psychology \\ Liverpool John Moore \\ University \\ Liverpool, United Kingdom \\ shfairclough@googlemail.com
}

\author{
Anton Nijholt \\ Human Media Interaction \\ University of Twente \\ Enschede, The Netherlands \\ a.nijholt@utwente.nl
}

\begin{abstract}
BCIs are traditionally conceived as a way to control apparatus, an interface that allows you to "act on" external devices as a form of input control. We propose an alternative use of BCIs, that of monitoring users as an additional intelligent sensor to enrich traditional means of interaction. This vision is what we consider to be a grand challenge in the field of multimodal interaction. In this article, this challenge is introduced, related to existing work, and illustrated using some best practices and the contributions it has received.
\end{abstract}

\section{Categories and Subject Descriptors}

H.5.2 [Information Interfaces and Presentation]: User Interfaces-Evaluation/Methodology, Input devices and strategies

\section{General Terms}

Algorithms, Measurement, Performance, Design, Reliability, Experimentation, Human Factors

\section{Keywords}

Brain-Computer Interface (BCI), Intelligent sensors, HumanComputer Interaction (HCI)

\section{INTRODUCTION}

A Brain-Computer Interface (BCI) is traditionally conceived as a way to control apparatus, an interface that allows you to "act on" external devices as a form of input

Permission to make digital or hard copies of all or part of this work for personal or classroom use is granted without fee provided that copies are not made or distributed for profit or commercial advantage and that copies bear this notice and the full citation on the first page. To copy otherwise, to republish, to post on servers or to redistribute to lists, requires prior specific permission and/or a fee.

ICMI'12, October 22-26, 2012, Santa Monica, California, USA.

Copyright 2012 ACM 978-1-4503-1467-1/12/10 ...\$15.00. control $[9,11,21]$. However, most Brain-Computer Interfaces (BCIs) do not provide a reliable and efficient means of input control and are difficult to learn and use relative to other available modes. We propose to change the conceptual use of "BCI as an actor" (input control) into "BCI as an intelligent sensor" (monitor). This shift of emphasis promotes the capacity of BCI to represent spontaneous changes in the state of the user in order to induce intelligent adaptation at the interface. BCIs can be increasingly used as intelligent sensors which "read" passive signals from the nervous system and infer user states to adapt Human-Computer Interaction (HCI), Human-Robot Interaction (HRI), or HumanHuman Interaction (HHI). This perspective on BCIs challenges researchers to understand how information about the user state should support different types of interaction dynamics, from supporting the goals and needs of the user to conveying state information to other users. What adaptation to which user state constitutes opportune support? How does the feedback of the changing HCI, HRI, and HHI affect brain signals? Many research challenges need to be tackled.

This article introduces the grand challenge of BCIs as intelligent sensors for enhancing HCI. The next section will provide the required background and will discuss the challenge of BCIs as intelligent sensors for enhancing HCI, HRI, and HHI. Next, in Section 3 the best practices on BCIs as intelligent sensors will be presented. First three operational BCIs as intelligent sensors will be discussed. Subsequently, a concise discussion on the contributions accepted for this grand challenge will be presented. We conclude this article with a brief conclusion in Section 4 .

\section{ON BCI AS INTELLIGENT SENSOR}

The current grand challenge poses that BCIs can be used as intelligent sensors to enhance HCI, HRI or HHI. However, what does this mean and what does this imply? To answer this question, we define three sub questions:

1. How do BCIs as intelligent sensors relate to conventional BCIs and how do they enhance interaction?

2. What would make these BCIs intelligent? and 


\section{How could such BCIs be realized?}

These three questions will be addressed in the next three subsections and, in parallel, the work presented during the grand challenge will be held against them.

\subsection{Types of BCIs and how they can enhance interaction}

In 1973, Jacques J. Vidal coined the expression BCI [19]. Let us start with two quotes from his milestone article: "Spectral content and correlation 'have been related to various emotional and behavioral states. ... To provide a direct link between the inductive mental processes used in solving problems and the symbol-manipulating, deductive capabilities of the computer, is, in a sense, the ultimate goal in man-machine communication." (p. 157 and 158). Vidal [19] (more or less) already identified the value of BCI and Zander and Kothe [21] suggested the following distinction in outputs that are directly derived from brain activity:

- Active BCI : Independent of external events, for controlling an application.

- Reactive BCI : Arising in reaction to external stimulation, which is indirectly modulated by the user for controlling an application.

- Passive BCI : Arising without the purpose of voluntary control, for enriching HCI with implicit information on the actual user state.

The current grand challenge addresses BCI as passive BCI, which enhances other (traditional) interaction modalities (e.g., mouse, keyboard, speech and gestures). However, such a combination of modalities was already described by, among others, Nijholt and colleagues [10].

\subsection{Towards intelligence!?}

In principle, the processing pipeline of BCIs as intelligent sensors is no different from that of active or reactive BCIs up to the phase where the signals are received [7]. From that point on the processing pipeline is, most likely, essentially different. Recently, there has been a vivid discussion on the definition of BCI, which is described in [9]. However, for reasons of brevity, in this article, we adopt the definition of Pfurtscheller and colleagues [11], who determined that a (conventional) BCI should satisfy the following criteria:

1. signals are recorded directly from the brain;

2. at least one recordable brain signal, which the user can intentionally modulate to effect goal-directed behavior;

3. real time processing; and

4. the user must obtain feedback.

BCIs as intelligent sensors, however, need only to satisfy the first and third criteria. The fourth criteria can be satisfied; but, this is not a (strict) requirement. Implicit feedback of which the user is unaware would be in line with the concept of BCIs as intelligent sensors (e.g., a subtle change in the interaction with other (artificial) agents). This leaves us with the second criteria, which excellently marks the difference between conventional BCIs and BCIs as intelligent sensors. In both cases, at least one recordable brain signal is needed.
However, in the case of BCIs as intelligent sensors no intentional modulation (i.e., altering brain activity) to achieve a certain goal is needed.

BCIs as intelligent sensors are a type of passive BCI that monitor a person by recording signals directly from the brain and processes them fast, preferably in real time. However, subsequently, no direct (conscious) goal has to be met (e.g., driving a wheelchair). As we will discuss next, this can relieve the true complexities underlying BCIs [5, 17]:

1. the definition of constructs;

2. their operationalization; and, subsequently,

3. their mapping upon the biosignals (e.g., EEG signals or other (peripheral) signals).

The outcome of the processing pipeline could be interpreted in terms of higher cognitive processes (e.g., the amount of attention $[8,20]$ ), unconscious processes [12]), or (even) social processes $[1,15]$. With such processes a discretization into a certain number of levels is not needed, the direction the process is going to can already be sufficient [3, 6]. Furthermore, it may even not be necessary to interpret patterns in EEG signals in terms of cognitive processes, a physiologically driven system could also serve the user $[15,18]$.

\subsection{Suggestions for their implementation}

Loosening the criteria of intentional modulation (cf. [11]) also introduces additional complexity and requires more intelligence than with conventional BCIs. If there is not a specific goal to be met, what then is the target to aim at? And how does this influence the signal processing and machine learning part of BCIs' processing pipeline? With BCIs as intelligent sensors, an interdisciplinary approach would be advisable; so, this would imply that all signals need to be processed in an integral framework. Recent advances in neuroscience support such an approach, as cognitive processes are distributed and, at best, (some) can only be partly localized [12]. Moreover, such localized approaches often fail in beauty when brought outside the lab into (the noisy) real world practice. Although signal processing and machine learning can rely on a thorough foundation [17], the high complexity introduced by a holistic approach will make it challenging.

Signal processing and machine learning for BCIs as intelligent sensors asks for new paradigms and unconventional approaches (cf. [7]). This grand challenge introduces some of them. Kyrgyzov and Souloumiac [8] present a processing scheme for adaptive artifact rejection from the EEG. Plass Oude-Boss and colleagues [13] propose to improve BCI performance after classification. De Sa [3] proposes a method to tackle the traditional problem of non-stationary EEG signals. To enable more robust BCIs as intelligent sensors, they suggest the use of a "control signal that depends intimately on what has already been transmitted, interpreted, and received". Most likely a combination of methods and techniques will be required to enable a successful holistic approach as is needed with BCIs as intelligent sensors.

\section{BEST PRACTICES}

This section presents the best practices on BCIs as intelligent sensors. In the first subsection, three operational BCIs as intelligent sensors, each originating from another domain, 
will be discussed as representatives of current state of the art best practices. In the second subsection, a concise discussion is presented on the contributions accepted for this grand challenge.

\subsection{The state of the art}

Although BCIs are rarely approached as intelligent sensors, there has already been work conducted on this. Here, we present three examples of BCIs as intelligent sensors. The domains of application are art, gaming, and office work, which nicely illustrates the breadth of application of BCIs as intelligent sensors.

An artistic example of the use of BCI as an intelligent sensor is the exposition Staalhemel ${ }^{1}$ created by Christoph de Boeck. Staalhemel is an interactive installation with 80 steel segments suspended over the visitor's head as he walks through the space. Tiny hammers tap rhythmic patterns on the steel plates, activated by the brain waves of the visitor who wears a portable BCI (EEG scanner). Thus, visitors are directly interacting with their surroundings, in this case an artistic installation. Although the visitors have a certain level of control and get a kind of feedback on their user state by variations in the generated sounds, the exact level of control on the generated sounds is not deducible due to the intrinsic variability already present in, and the influence of other visitors on the sounds generated.

In Alpha-World of Warcraft (alphaWoW) [14] neural correlates with the mood of players are coupled to their avatars in the immersive game environment. Alpha activity (8-12 $\mathrm{Hz}$ rhythms in the EEG) recorded over the parietal lobe is used to control one aspect of the game character, while conventional controls are still used for the rest. A user playing World of Warcraft ${ }^{\circledR}$ can use both conventional controls and brain activity to control her character in the game. How changes in alpha activity are experienced by the user, depends on the location where the activity is measured. According to Cantero et al. [2], high alpha activity measured over the parietal lobe is related to a relaxed alertness. This seems a beneficial state of mind for gaming, especially compared to drowsiness, which is said to be measured frontally.

Transparant is an office window that aims to reduce distractions in the user's environment by varying its opacity based on the user's level of focus [4]. The state of the user (e.g., this focus) is determined by a wireless EEG headset, which communicates the user state to a smart glass module which changes the transparency of the office window accordingly. The opacity of the office window is based on the state of the user and changes accordingly. This serves two goals: i) support to be and remain focussed and ii) a signalling function whether or not the user can be disturbed.

\subsection{Contributions to this grand challenge}

This subsection takes the article of Fairclough and Gilleade [5] as starting point and adopts their biocybernetic loop as fundamental and generic to all categories of physiological computing system, including BCI. The biocybernetic loop describes the data processing protocol at the heart of all physiological computing systems, including BCI. The loop also encompasses the goals of the system design with respect to the anticipated impact of the adaptation on user behaviour. Par excellence, it supports the positioning of the contributions of the current grand challenge. The article

\footnotetext{
$\overline{{ }^{1} \text { http://www.staalhemel.com/ }}$
}

by Ramirez and Vamvakousis [16] is concerned with the use of EEG classification to transform the audio properties of musical performance. In this case, the emotional responses from the performer are translated into a model of emotional states. The authors describe how EEG measures may be interpreted within an activation-valence space to describe emotional responses. The next step would be to translate those emotional responses into appropriate audio transformations, that is, audio manipulations that represent and possibly reinforce the emotional state of the performer.

A number of articles focused on the inherent challenges of stage four when the system must classify the psychophysiological data into specific categories in order to trigger a response. Plass-Oude Bos and colleagues [13] describe simple methods to improve detection accuracy after the incoming brain activity has already been classified, such as gathering additional evidence from other sources of information, for instance context, and transforming the unstable classification results to be more easy to control. They provide an overview of the different techniques, showing where to apply them and comparing the effects.

Kyrgyzov and Souloumiac [8] present an approach to improve system interaction via EEG by refining the process of artifact rejection. The EEG is especially susceptible to the influence of gross movement, such as head movement, as well as the impact of eye movement on frontal sites. This adaptive approach to artifact rejection, which involves precalibration of the user and independent component analysis, represents a way of reducing the number of erroneous responses from the system.

The article by de Sa [3] presents the intriguing possibility of categorizing user perceptions of what constitutes an appropriate response into the process of classification. The biocybernetic loop generally represents a mapping of psychophysiological triggers onto commands elicited at the interface, for example move up, move down. These authors argue that EEG activity could be classified into categories of intended and unintended movement; this distinction could be used to guide input control and allow the system to detect erroneous movement.

Physiological computing systems have the potential to increase the efficiency of human information processing as well as providing novel methods for communication. The article by Weiden and colleagues [20] describes how detection of the P300 component may be used to improve the classification of moving images based on rapid presentation. This technique was previously used with still images but the application of this technique to moving images is novel.

The contribution from Pope and Stephens [15] is concerned with the application of biofeedback training across individuals in order to create a collective dynamic, that is, two users collaborating either competitively or as part of a team. This paper describes how physiological responses may be aggregated and modulated on a group basis. This type of system relies heavily on the latter stages of adaptive design and evaluation. The papers presented in the Grand Challenge have focused on improvement of classification and the implementation of novel application domains.

\section{CONCLUSION}

This paper has focused on what we consider a grand challenge in BCI: the use of a users' physiological signals to automatically adapts HCI. This grand challenge has signif- 
icant implications for BCI, HCI, HRI, HHI, as well as for neuroscience. It asks for a new definition of BCI and a (significant) adaptation of its processing pipeline (cf. [9]). HCI can be enriched in subtle ways and interactions hinting towards intelligence become within reach. Moreover, par excellence, it could provide neuroscience the means to validate its lab research in real world contexts. Hence, BCIs as intelligent sensors can add to basic knowledge on neuroscience, requires rethinking signal processing and machine learning paradigms for BCI, can enrich HCI, and, as such, yields a new landscape of BCI applications orthogonal to many of the existing ones.

If anything, this grand challenge brought, once more, to bear that a true interdisciplinary approach is required in making BCIs as intelligent sensors a success. A prerequisite for this requirement is not only close cooperation between disciplines, which yields multidisciplinary approaches, but true interest in and respect for each others theories, methods and models. Then, and only then, can true interdisciplinary research arise. If this can be achieved, the development of BCIs as intelligent sensors can and will take a leap forward.

\section{ACKNOWLEDGMENTS}

The authors Nijboer, Nijholt and Poel gratefully acknowledge the support of the BrainGain Smart Mix Programme of the Netherlands Ministry of Economic Affairs and the Netherlands Ministry of Education, Culture and Science.

\section{REFERENCES}

[1] R. Adolphs. Cognitive neuroscience of human social behaviour. Nature Reviews - Neuroscience, 4(3):165-178, 2003.

[2] J. L. Canteroa, M. Atienzaa, C. M. Gómez, and R. M. Salasa. Spectral structure and brain mapping of human alpha activities in different arousal states. Neuropsychobiology, 39(2):110-16, 1999.

[3] V. R. de Sa. An interactive control strategy is more robust to non-optimal classification boundaries. In Proceedings of the 14th international conference on multimodal interfaces, ICMI '12, 2012.

[4] A. Ducao, T. Tseng, and A. von Kapri. Transparent: Brain computer interface and social architecture. In Proceedings of the SIGGRAPH 2012, 2012.

[5] S. Fairclough and K. Gilleade. Construction of the biocybernetic loop: A case study. In Proceedings of the 14th international conference on multimodal interfaces, ICMI '12, 2012.

[6] J. H. Janssen, E. L. van den Broek, and J. H. D. M. Westerink. Tune in to your emotions: A robust personalized affective music player. User Modeling and User-Adapted Interaction, 22(3):255-279, 2012.

[7] D. J. Krusienski, M. Grosse-Wentrup, F. Galán, D. Coyle, K. J. Miller, E. Forney, and C. W. Anderson. Critical issues in state-of-the-art brain-computer interface signal processing. Journal of Neural Engineering, 8(2):025002, 2011.

[8] O. Kyrgyzov and A. Souloumiac. Adaptive EEG artifact rejection for cognitive games. In Proceedings of the 14th international conference on multimodal interfaces, ICMI '12, 2012.

[9] F. Nijboer, J. Clausen, B. Z. Allison, and P. Haselager. The asilomar survey: Stakeholders' opinions on ethical issues related to brain-computer interfacing. Neuroethics, pages 1-38, 2011.

[10] A. Nijholt, D. P.-O. Bos, and B. Reuderink. Turning shortcomings into challenges: Brain-computer interfaces for games. Entertainment Computing, 1(2):85-94, 2009.

[11] G. Pfurtscheller, B. Z. Allison, C. Brunner, G. Bauernfeind, T. Solis-Escalante, R. Scherer, T. O. Zander, G. Mueller-Putz, C. Neuper, and N. Birbaumer. The hybrid BCI. Frontiers in Neuroscience, 4:Article No. 30, 2010.

[12] C. L. Philippi, J. S. Feinstein, S. S. Khalsa, A. Damasio, D. Tranel, G. Landini, K. Williford, and D. Rudrauf. Preserved self-awareness following extensive bilateral brain damage to the insula, anterior cingulate, and medial prefrontal cortices. PLoS ONE, 7(8):e38413, 2012.

[13] D. Plass Oude-Bos, H. Gürkök, B. Reuderink, and M. Poel. Improving BCI performance after classification. In Proceedings of the 14th international conference on multimodal interfaces, ICMI '12, 2012.

[14] D. Plass-Oude Bos, B. Reuderink, B. van de Laar, H. Gürkök, C. Mühl, M. Poel, A. Nijholt, and D. Heylen. Brain-computer interfacing and games. In D. Tan and A. Nijholt, editors, Brain-Computer Interfaces. Applying our Minds to Human-Computer Interaction, Human-Computer Interaction Series, pages 149-178. Springer Verlag, London, 2010.

[15] A. Pope and C. Stephens. Interpersonal biocybernetics: Connecting through social psychophysiology. In Proceedings of the 14th international conference on multimodal interfaces, ICMI '12, 2012.

[16] R. Ramirez and Z. Vamvakousis. Emotion-based EEG control in music performance. In Proceedings of the 14 th international conference on multimodal interfaces, ICMI '12, 2012.

[17] E. L. van den Broek. Affective computing: A reverence for a century of research, volume 7403 of Lecture Notes in Computer Science, pages 434-448. Berlin/Heidelberg, Germany: Springer-Verlag, 2012.

[18] E. L. van den Broek, J. H. Janssen, J. H. D. M. Westerink, and J. A. Healey. Prerequisites for Affective Signal Processing (ASP). In P. Encarnação and A. Veloso, editors, Biosignals 2009: Proceedings of the International Conference on Bio-Inspired Systems and Signal Processing, pages 426-433, Porto - Portugal, 2009.

[19] J. J. Vidal. Toward direct brain-computer communication. Annual Review of Biophysics and Bioengineering, 2(1):157-180, 1973.

[20] M. Weiden, D. Khosia, and M. Keegan. Electroencephalographic detection of visual saliency of motion towards a practical brain-computer interface for video analysis. In Proceedings of the 14th international conference on multimodal interfaces, ICMI '12, 2012.

[21] T. O. Zander and C. Kothe. Towards passive brain-computer interfaces: Applying brain-computer interface technology to human-machine systems in general. Journal of Neural Engineering, 8(2):025005, 2011. 